\title{
Quacksalber und Kurpfuscher in den deutschschweizerischen Kalendern des 19. Jahrhunderts*
}

\author{
Von Barbara C.Hansch-Mock
}

Der Sinn meiner Untersuchung bestand darin, das schul- und volksmedizinische Material, wie es in den Kalendern des 19. Jahrhunderts der deutschsprachigen Schweiz erscheint, zu untersuchen. Das Quellenmaterial bot die umfangreiche Sammlung schweizerischer Volkskalender des Seminars für Volkskunde an der Universität Basel. Die Herausgeber und Redaktoren dieser Kalender wendeten sich an weite Kreise der Bevölkerung. Wenn sie medizinische Gegenstände aufnahmen, so wollten sie damit zur Verbreitung bestimmter Kenntnisse beitragen. Der Laie sollte vor allem seine Krankheit erkennen und diese mit einfachen, jedermann zugänglichen Hilfsmitteln kurieren lernen. Hierzu wurden Rezepte sehr verschiedenen Ursprungs geboten. Standorte von Heilpflanzen werden genannt, ferner die Herstellung von Medikamenten mit den dazu benötigten Instrumenten; erläutert werden das Pro und Kontra gebräuchlicher Heilkuren, Probleme der Diätetik, Hygiene und Krankenpflege. Zu Beginn der fünfziger Jahre kamen - im Zuge der Zeit - noch medizinische Inserate dazu.

Es besteht kein Zweifel, daß sich die Kalender beim «Volk» großer Beliebtheit erfreuten, allein schon, weil sonstige Bücher schwer zugänglich waren, von der Bibel und fliegenden Blättern abgesehen. Die volksmedizinischen Mittel zur Prophylaxe und Therapie, zusammen mit den vielen hygienischen Regeln aus dem 19. Jahrhundert, sind zuverlässige Fakten der Volksmedizin und der Medizingeschichte. Kurz : die Kalender registrieren und popularisieren zugleich das volksmedizinische Wissen ihrer Zeit.

Ein besonders beliebtes Thema der Kalenderschreiber war das der Quacksalber und Kurpfuscher. In der Schweiz finden wir vom 17. Jahrhundert an gesetzliche Bestimmungen gegen Quacksalber und Marktschreier ${ }^{1}$, die aber immer wieder unbeachtet blieben. Laut Hunziker ${ }^{2}$ waren 1914 in der Schweiz noch 189 notorische Kurpfuscher bekannt. Heute gilt immerhin in den meisten Kantonen das Verbot der ärztlichen Tätigkeit durch Laien ${ }^{3}$.

* Auszug aus der volkskundlichen Dissertation «Deutschschweizerische Kalender des 19. Jahrhunderts als Vermittler schul- und volksmedizinischer Vorstellungen», die in der Reihe "Veröffentlichungen der Schweizerischen Gesellschaft für Geschichte der Medizin und der Naturwissenschaften» im Verlag Sauerländer, Aarau, erscheint. 
Auf scherzhafte Weise versucht folgende Anekdote im «Zürcher Kalender » von 1849 den Mangel an Vertrauen zum Arzt zu zeigen. Von einer armen Frau, die auf ihrem letzten Lager liegt, heißt es: «Sie ist zu arm zum Doktern und für den Tod ist kein Kraut gewachsen. Doch wird's ihr alle Tage änger und bänger, und sie läßt den Arzt bitten, er möge doch der Gotteswillen zu ihr kommen; vielleicht könne er ihr's ein wenig leichter machen.» Darauf folgt die charakteristische Auffassung: «Ich glaube bestimmt, wenn sie den Vetter Doktor früher beschickt hätte, so hätte sie schon sterben können.» Kein Jobenswertes Verhalten des Arztes zu seinem Patienten geht aus folgendem Beispiel hervor, welches dem «Zürcher Kalender» von 1851 entnommen wurde. Hier bittet ein Bauernbub den Arzt, sofort zu kommen, da sein Vater einen Knochen verschluckt habe und sonst ersticken würde. "Nachdem eine halbe Stunde verstrichen ist, fragt ein Dritter: 'Herr Doktor, warum gehn Sie denn nicht?' 'Sehn Sie, der Bube hat eine Stunde hereingebraucht und ich brauche solange hinaus. Ist der Bauer nun in zwei Stunden nicht erstickt, so erstickt er auch nimmer, ist er aber erstickt, so kann ich nichts mehr helfen. Ich bin also in jedem Falle überflüssig.' » Das Bild des negativ eingestellten und egoistischen Arztes, der jede körperliche Anstrengung und Störung der nächtlichen Ruhe und Gemütsbewegung mied, war im Volk sehr verbreitet und wurde durch solche und viele ähnliche Kalendergeschichten gefördert. Es war keine Neuigkeit, daß die «wahrhaft guten Ärzte, die redlich und ohne eigenes Interesse das Heil der leidenden Menschheit fördern, zu den Ausnahmen» zählten, und die «eigennützigen die Regel ausmachen ». ${ }^{4}$ So ist es nicht verwunderlich, daß mancher mit solchen Ansichten sich den Händen des Quacksalbers auslieferte.

Vertreter aller Berufsschichten, Stände und Volksschichten haben sich als Heilkünstler betätigt. Auch die Kalender geben Aufschluß über die Leute, die sich als Quacksalber übten. So sind es z. B. «alte, geschwätzige Weiber, Schmiede und Viehdoctoren», wie der «Wirtschaftskalender des aufrichtigen und wohlerfahreren Boten» von 1830 aus Aarau berichtet. In einem elfstrophigen Gedicht warnt der «Lustige Schweizer» aus Schaffhausen 1845 unter dem Titel «Der Wunderdoktor, ein Märchen aus unserer Zeit» vor den Kurpfuschern, die früher Schuhknechte waren: «Nun höret eine Mähre an, gar wunderbar zu lesen. Im Klettgau wohnt ein Wundermann, einst Schuhknecht nur gewesen. Den hat der Geist so inspiriert, daß er zum Doktor avanciert - in Gerbermeisters Bade.»

Der Kampf gegen das Kurpfuschertum vollzog sich zusammen mit der Verbreitung der allgemeinen medizinischen Aufklärung. Die Kalender wiesen schon seit frühester Zeit auf die bösen Folgen jener Ratschläge hin. Sie warnten vor «Betrügern und Mördern, die ihren Nächsten aus Gewinnsucht um Leben und 
Gesundheit bringen. ${ }^{5}$ Allmählich wird in den Kalenderartikeln der Unterschied deutlich zwischen Kurpfuschern und Quacksalbern. Dem Kurpfuscher wurden in seinen Heilmethoden ehrliche Verfahren zugetraut; aber aus Mangel an medizinischen Kenntnissen bediente er sich falscher Mittel. Der Quacksalber und Marktschreier hingegen wird tiefer eingestuft, denn er sei sich der Untauglichkeit seiner Methoden und Heilmittel bewußt, baue aber skrupellos auf die Leichtgläubigkeit der Menschen ${ }^{6}$. Er will den Kranken ausbeuten und wirbt durch Heilversprechen, Bluff und Großtuerei. Wenn bei den Quacksalbern tatsächlich Heilung vorkommt, so geschieht dies meistens durch Suggestion. Oft waren ihre Mittel harmlose farbige Wässerlein oder Brotkügelchen. Der Luzerner «Neue Hauskalender» von 1820 berichtet von zwei Schelmen, die mit dem Verkauf von harten Brotkügelchen als Zahnwehmittel ihren Lebensunterhalt verdienten. Die Geschichte verläuft so, daß einer in die Wirtschaft geht, sich krank stellt und vor Zahnschmerzen laut jammert. Bald darauf kommt «zufällig» sein Freund, als Arzt verkleidet, daher, verspricht, ihn von seiner Qual zu befreien, indem er sein prächtiges und wirkungsvolles Mittel lobt und dem Patienten eins davon schenkt. Dessen Schmerzen sind blitzartig verschwunden, und nun wollen natürlich alle Gäste von diesem Wundermittel beziehen, und der Betrüger macht ein gutes Geschäft. Die warnende Schlußfolgerung des Kalenderschreibers lautet: «Jedem, der Pfuschern glaubt, ... geht es so.» ${ }^{7}$

Außer mit mehr oder weniger harmlosen Mitteln arbeiteten die Quacksalber häufig mit ganzen Dreckapotheken und, was noch schlimmer war, mit schädlichen Giften. Besonders beliebt waren auch alle Arten von Tierfetten, dem von Bären, Hasen, Löwen, Schlangen, Katzen und Hunden, ferner Spinngewebe, Haare, Pferdeschweiß, Mäusedreck, Heringe, Ohrenschmalz, Kellerasseln, Mehlwürmer usw. ${ }^{8}$

An Hand der Kalender lassen sich Bilder einiger markanter Persönlichkeiten erkennen, die in der Schweiz mit großem Erfolg ihr Handwerk betrieben. Als einer der berühmtesten Pfuscher galt Michael Schüppach (1707-1781), genannt Schärer Micheli, von Langnau i.E. Von ihm weiß man, daß Goethe ihn einmal besucht hat ${ }^{9}$. Dem Bauerndoktor widmen verschiedene Kalender lange rühmende, ehrende und sympathische Artikel. So bringt der «Schweizer Bote» (Aarau 1828) unter dem Titel «Biographie merkwürdiger Männer » nicht weniger als $6 \frac{1}{2}$ Seiten über den Wundertäter ${ }^{10}$ : "Vornehme Leute kamen aus allen Ländern um seine Hilfe in Anspruch zu nehmen, denn er war Mode. Auf seinen Büchsen und Salbentöpfen waren wunderliche Namen angebracht wie: Freudenöl, Prophetenbeeri, Blümliherz, Maria-Theresia-Öl, Kreuzhölzlisalat.» Als positive Verordnung soll er Schwelger und Prasser, besonders ausländische, dadurch geheilt haben, daß 
er ihnen frugale Schweizerkost empfahl. Außer diesen klugen Vorschlägen brauchte er primitive, doch meist unschädliche Mittel.

Ein bekannter Wunderarzt, der um die Mitte des 19. Jahrhunderts lebte und seinen Sitz in «Rebstein», dem ehemaligen Schloßgebäude des Fürstabtes von St. Gallen im St. Galler Rheintal, hatte, war Georg Blatter. Die Leute kamen zu ihm aus St.Gallen, Vorarlberg, Schwaben, dem Elsaß, Graubünden, Appenzell und Liechtenstein. Neben seinem Beruf als Siebmacher hatte er sich noch weitere Titel angeeignet: Wundertäter, Nigromant, Teufels- und Hexenbeschwörer, Gespensterbanner, Herbeischaffer gestohlener Sachen, Kundiger des Erdspiegels und der Wünschelrute und Besitzer aller geheimen Schriften von Paracelsus und Albertus Magnus. So berichtet der «Thurgauer Volksfreund»1867, daß Blatter seine höchsten Triumphe im Jahre 1865 feierte. Selbst Bischöfe kämpften vergeblich gegen ihn. Er hatte großen Zulauf; nicht selten behandelte er achtzig Personen an einem Tag. Doch das Unumgängliche stellte sich bald heraus: seine Heilungen waren nicht von langer Dauer, und somit schwand auch die Begeisterung des Volkes dahin. Alles hatte bei ihm aus Selbsttäuschung oder einer «Besserung von vorübergehender Dauer» bestanden.

Von einem anderen Stümpelarzt berichtet der «Lustige Schweizer» 1817. Dies war der Vieharzt Peter Zofinger, der als Knabe Schäfer war und das Schreiben nie gelernt hatte. Laut Kalenderangaben zweifelte das Volk nicht an seiner Behauptung, er habe eine besondere Gottesgabe zu heilen, er sei weit durch Ägypten und die Wüste gereist, habe viele Wunderkuren gemacht und unzählige Ordenskreuze erhalten. Der Verfasser des Kalenders berichtet, «es könnten noch hundert Böke von diesem Wunderdoktor erzählt werden ». Beim Beschauen eines Knabenurins soll er beispielsweise gesagt haben: «Dem Mädchen soll man einen Mann geben », oder ein anderes Mal muß er empfohlen haben, daß man einem Patienten die «Leberader» am linken Arm öffnen solle, «wie wenn es dort eine Leberader gäbe». Das Pfuschen wurde ihm vom Kantonsrat von Schaffhausen sowie von der badischen Regierung verboten.

Aus dem zitierten Kalendermaterial mag hervorgehen, daß die Verfasser versucht haben, die Quacksalber als Betrüger und unehrenhafte Gesellen dem Volk darzustellen. Eine Ausnahme war Michael Schüppach, der bei den Kalenderschreibern hohes Ansehen genoß und noch «heute in lebhafter Erinnerung der Bevölkerung des Emmentals steht». ${ }^{11}$ Aufklärerische Versuche gehen durch das ganze 19. Jahrhundert hindurch und werden um so energischer, je mehr sich die Pfuscher die Leichtgläubigkeit der Masse zunutze machen. Gerade aus diesem Grund scheint es um so verwunderlicher, daß sich trotz aller Fortschritte der Medizin Abarten des Pfuschertums und andere Irrlehren bis heute erhalten konn- 
ten und wieder zunehmen. Es scheint, daß das 20. Jahrhundert in dieser Hinsicht wieder mehr Nachsicht gewähren will ${ }^{12}$.

Der Verkauf und Unfug, der mit den geheimgehaltenen Arzneimitteln getrieben wurde, beschäftigte die Kalender in der zweiten Hälfte des 19. Jahrhunderts. Nicht nur Apotheker und Ärzte trieben solchen Handel; sondern auch Laien aus allen Berufsschichten erklärten sich für kompetente Heilkundige und Händler ${ }^{13}$. Neben solchen Einzelpersonen waren es hauptsächlich Apotheker, die Arzneimittel in der ganzen Schweiz verschickten. Aber auch «anerkannte» Institutionen wie etwa «Britische-Gesundheits-Colleges» (Appenzeller Kalender 1888), die «Pariser Akademie de Medicine» (St. Galler Kalender 1886) oder das «internationale Etablissement für arzneilose Heilkunst» (Eidgenössischer Dorfkalender 1899) wollten beim Geschäft mit Geheimmitteln dabei sein.

Wenn die Kalender erstmals in den vierziger und fünfziger Jahren medizinische Inserate zu Propagandazwecken erscheinen lassen, so geschieht dies aus spekulativen Gründen. Als fragwürdiger Pionier in dieser Hinsicht darf der «Eidgenössische National Kalender» von 1845 gelten; darauf folgen der «Appenzeller Kalender» 1846, der «Zürcher Kalender» 1847, der «Schweizerische Dorf kalender» 1858 und «Der Prophet» 1858. Gegen Ende des Jahrhunderts nimmt der Propagandateil oft beträchtlichen Umfang an. Die neue Einnahmequelle war beachtlich, denn entweder verlangten die Verleger hohe Preise für die Inserate, oder sie priesen Mittel an, die sie selbst fabrizierten und verkauften. In einer «Warnung vor Geheimmitteln» versucht der Herausgeber des «Schaffhauser Boten» 1898, das Erscheinen der Schwindelpropaganda am Schluß des Kalenders zu rechtfertigen: «Der Kalendermann schaut mit Bangen auf die vielen Anpreisungen, welche hinten an seinen Kalendergeschichten stehen. Er hat sie nicht hindrucken lassen; es steht auch nicht in seiner Macht, dieselben zu mustern und hinauszuwerfen, was ihm nicht gefällt.» Er bittet die Leser, ihn für ein gefehltes Inserat nicht verantwortlich zu machen, und gibt den Ratschlag: «Seid auf der Hut!» Offenbar brachten auch Tageszeitungen, um ihre Ehre zu retten, ähnliche Zeilen: es sei ganz unmöglich, daß eine Redaktion, welche Einsendungen erhalte, wissen könne, wer ehrlich oder unehrlich, was giftig oder ungiftig sei ${ }^{14}$.

Hinsichtlich solcher Inserate für Geheimmittel lassen sich einige charakteristische Züge feststellen: Die Patienten müssen nur ihre Symptome notieren, und schon erfolgt brieflich die Nennung entsprechender Mittel. Die «hohe Qualität» solcher Produkte wird folgendermaßen belegt: Die Arznei ist jeweils von Ärzten «beglaubigt»; Hunderte von Zeugnissen zufriedener Patienten in ganz Europa attestieren die Heilung; die Mittel hatten fast immer goldene Medaillen und Auszeichnungen erhalten oder waren auf Weltausstellungen preisgekrönt worden. 
Daß ausländische Produkte mehr gelten als einheimische, machten sich die Verfasser solcher Inserate zunutze. Wundermittel stammen aus Österreich, Leipzig, Westfalen, Weimar, Amsterdam und London. Auch wurden für die Benennung einzelner Mittel gern fremde Sprachen angewendet. Besonderer Beliebtheit erfreuten sich Latein und Englisch, wie folgende Beispiele zeigen: «Arcanum miraculosum», ein Schönheitsmittel, «Safe-Cure», «Pain-Expeller», «Pain-Killer» gegen Rheuma und «Auxilium orientis» gegen Epilepsie. Bemerkenswert ist der «Einsiedler Kalender», der von 1857 bis 1871 im Inseratenteil ganze Erklärungen über Wirkung und Anwendung einzelner Mittel in englischer Sprache bringt. Aber auch in deutscher Sprache wird auf ausländischen Ursprung der Ware hingewiesen, in der richtigen Annahme, das Publikum sei fremdländischem Einfluß besonders zugänglich: «Schwedische Lebensessenz», «Orientalisches Enthaarungsmittel», «Indischer Extrakt» gegen Flechten und Zahnweh, «Chinesisches Haarfärbemittel», «Russischer Frostbalsam», «Amerikanisches Öl» gegen Geschlechtsleiden und englische oder ungarische Haarerzeugungsmittel.

Wenn der Textteil der Kalender sich größtenteils mit der Heilung von alltäglichen Leiden (Warzen, Wunden, Kopfweh) zufriedengibt, beschäftigt sich der Inseratenteil mit schwer heilbaren Krankheiten wie Gehirnleiden, Epilepsie, Bettnässen oder Haarausfall. Die im Textteil nie erwähnten «geheimen Krankheiten », die «durch Jugendsünden entstanden oder durch Ansteckung erworben sind», wie Geschlechts- und Frauenkrankheiten, Impotenz, Trunksucht, werden hier «streng diskret und promt brieflich geheilt».

Vergleicht man das medizinische Kalendermaterial mit den Ergebnissen der im Schweizerischen Institut für Volkskunde vorhandenen Volksumfragen ${ }^{15}$, so geht daraus hervor, daß die Kalender doch bedeutenden Einfluß auf ihre Leser hatten. Es besteht freilich die andere Möglichkeit, daß die Kalender «volkstümliches Wissen » verwertet haben. Andererseits hat sich aber eine starke literarische Bindung der medizinischen Ratschläge deutlich ergeben. Anleihen bei den volkstümlichen Vorstellungen sind eher gering. Somit erklären sich die Parallelen doch zur Hauptsache über die Rezeption der «Kalenderweisheit».

Daß sich der eigentliche Zweck der Kalender im Laufe des 19. Jahrhunderts gewandelt hatte, wußten die Verfasser selber zu berichten. Der «Zürcher Kalender» von 1880 schreibt: «Früher suchte man in Kalendern gemeinnützige Anregungen in Haus und Feld, für Familie und Vieh, und verwendbare Rezepte und Anweisungen. 'So sei der Kalender wenigstens unterhaltend', sagen die Leute, 'es ist uns sogar lieber, denn über dem Lesen der belehrenden Kalender schlafen wir ein.'» Gegen Ende des 19. Jahrhunderts hatten die Kalender sicher nicht 
mehr die gleiche Bedeutung, die ihnen damals zukam, als weder Zeitung noch Bücher zu erschwinglichen Preisen für jedermann erhältlich waren. Trotzdem gibt es auch heute noch Volkskalender. Der Zweck ihres Daseins hat sich allerdings verändert. Neben ihrer Funktion als Agenda ist ihre wichtigste Aufgabe: sie sollen unterhalten. Kalender mit eigentlich medizinischer Zielsetzung ${ }^{16}$ konnten sich immerhin bis weit ins 20. Jahrhundert hinein erhalten. Ob nun bei der heutigen Fülle an populärmedizinischer Fachliteratur, aufklärenden Sendungen

am Radio und Fernsehen und bei der wachsenden Zahl von Ärzten es völlig überflüssig oder gar absurd wird, für medizinische Ratschläge den Kalender aufzuschlagen, wie es in der vergangenen Epoche, mit der wir uns befaßt haben, üblich gewesen ist, bleibt abzuwarten ${ }^{17}$. Auch heute gilt der Aufruf zum ernsthaften Studium der Geschichte der Volksmedizin, deren Beurteilung «Kriterien für die heutige Situation beisteuern könnte».18

\section{Anmerkungen}

1 H. Magnus, Das Kurpfuscherthum, S. 24-25.

$2 \quad$ Hunziker, Der Kampf gegen das Kurpfuschertum in der Schweiz, S. 8.

3 Ausnahmen bilden nur die Kantone Appenzell-Außerrhoden und Graubünden.

4 G.F.Most, Encyclopädie, III, S. 433.

5 H.Kohlbecker, Allgemeine Entwicklungsgeschichte des badischen Kalenders ..., Diss. Freiburg i. Br., S. 20.

6 Vgl. E.Lombard, S.120; C. Müller, Jeremias Gotthelf und die Ärzte, S.119.

7 Eine ähnliche Geschichte bringt J.P.Hebel im «Rheinländischen Hausfreund» von 1807. Vgl. auch J.P. Hebel, Erzählungen des Rheinländischen Hausfreundes, I, Insel-Verlag, Frankfurt 1968, S.164.

8 G. Kersten, Die beiden Gesichter der Medizin, S.95.

9 H.A.Meyenberg, Kurpfuscherei, S.11.

10 Vgl. auch «Der Lustige Schweizer» (Schaffhausen) 1818, «Der Schweizerische Volksfreund» (Schaffhausen) 1866 und «Der Volksboten Schweizer Kalender» (Basel) 1882.

11 L. Baechler, Schweizerische Volkskalender, S.7.

12 G.Schindelholz, Grimoires secrets, Porrentruy 1973, bezeugt 25 Heiler in katholischen Gebieten des Juras, die göttliche Heilkraft haben sollen.

13 H.A.Meyenberg, Kurpfuscherei, S.22-23: In der Liste von Berufsarten finden sich u. a. Pfarrer, Verleger, Kaufmann, Polizist, Architekt, Gipsermeister, Redaktor, Masseur, Lehrer, Kutscher.

14 J. L. Sonderegger, Geheimmittelmarkt, S. 31.

15 Die Volksmedizinische Sammlung im Schweizerischen Institut für Volkskunde, Basel, besteht aus Enquête I (1934), dem Ergebnis einer Umfrage 
über Volksmedizin in der Schweiz », ferner aus volksmedizinischem Material von G. A. Wehrli (Dr. med. ZH), das 1910 bis 1920 zusammengetragen wurde, und handschriftlichem Material, welches von Frau Dr. M. Möckli-v. Seggern geordnet und ergänzt wurde.

16 z. B. Blaukreuz-Kalender, Rotkreuz-Kalender und Pfarrer-Künzle-Kalender. Die 1877 gegründete Blaukreuz-Bewegung setzte sich hauptsächlich die Bekämpfung des Alkoholismus zum Ziel.

17 R.Schenda, Volksmedizin, S.192. Ruth Friedrich, «Allgemein menschliches Verhalten des Großstädters», in: Beiträge zur Deutschen Volks- und Altertumskunde, Bd.15, 1971, S.61 ff.: Hier werden Personen aufgeführt, deren volksmedizinisches Verhalten sich auch heute noch kaum von dem ihrer Vorfahren unterscheidet.

R.Schenda, Volksmedizin, S. 192.

\section{Bibliographie}

Baechler, L., Aus dem medizinischen Inhalt schweizerischer Volkskalender im 18. und 19. Jahrhundert, in: Schweizer Hausapotheke, Jg. 14, Nr. 1, Januar 1943.

Friedrich, Ruth, «Allgemein menschliches Verhalten des Großstädters », in: Beiträge zur Deutschen Volks- und Altertumskunde, Bd.15, 1971.

Hebel, Johann Peter, Erzählungen des Rheinländischen Hausfreundes, hrsg. von Eberhard Meckel, Bd.I, Frankfurt 1968.

Hunziker, Der Kampf gegen das Kurpfuschertum in der Schweiz, Sonderabdruck aus: Veröffentlichungen aus dem Gebiete der Medizinalverwaltung, Bd.27, Heft 8, Berlin 1928.

Kersten, G., Die beiden Gesichter der Medizin, Hamburg 1903.

Kohlbecker, Hellmut, Allgemeine Entwicklungsgeschichte des badischen Kalenders in der Zeit von 1700 bis 1840, Diss. Freiburg i. Br. 1928.

Lombard, Eduard, Der medizinische Inhalt der schweizerischen Volkskalender im 18. und 19. Jahrhundert, Diss. Zürich 1925.

Magnus, H., Das Kurpfuscherthum, Breslau 1903.

Meyenberg, Heinrich Anton, Kurpfuscherei zu Jeremias Gotthelf Zeiten und Heute, Diss. Bern 1954.

Most, Georg Friedrich, Encyclopädie der gesammten medizinischen und chirurgischen Praxis, Bd.1-4, Leipzig 1843.

Müller, Carl, Jeremias Gotthelf und die Ärzte, Bern 1959.

Schenda, Rudolf, Volksmedizin - was ist das heute ? in : Zeitschrift für Volkskunde 69 (1973) II, 189-210.

Schindelholz, Georges, Grimoires secrets, Porrentruy 1973.

Sonderegger, Jakob Laurenz, Der Geheimmittelmarkt, St. Gallen 1880. 
Summary: Quacks in the Swiss-German Almanacs of the 19 th Century

When the editors of these almanacs took up the subject of medicine and especially that of the quacks, it was their goal to reach large parts of the population. In Switzerland we find regulations against quackery from the 17 th century on. The picture of the egoistic or selfish doctor, who strictly avoided all stress and inconveniences, was widely spread among the people and was promoted by countless articles throughout the almanacs. Therefore, it wasn't at all surprising that many landed in the hands of a quack. The fight against quackery was initiated in connection with the general enlightenment of medicine at that time. The almanacs inform us of the professions they had and their often times harmful medicine. We get to know some of the prominent figures who were popular. Advertising appears for the first time in the 1950's out of speculative reasons.

Dr. phil. Barbara C. Hansch-Mock

Birsigstraße 127

4054 Basel 